\title{
EL CENTRO HISTÓRICO DE LIMA ENTRE CONSERVACIÓN Y USO INADECUADO DEL PATRIMONIO HISTÓRICO ARQUITECTÓNICO 'MENOR'
}

\author{
THE HISTORIC CENTER OF LIMA: CONSERVATION VERSUS INAPPROPRIATE USEOF 'MINOR' \\ ARCHITECTURALHERITAGE
}

ANGELA LOMBARDI ${ }^{2}$ / PATRIZIA MONTUORI ${ }^{3}$

Fecha de recepción: 25 de setiembre de 2014.

Fecha de aprabación: 26 de noviembre de 2014.

\section{RESUMEN}

El casco antiguo de Lima es un patrimonio histórico-artístico en precarias condiciones de conservación que necesita una adecuada y firme acción de protección. Este artículo desarrolla una ponencia presentada en Lima en las V Jornadas de Conservación y Restauración organizadas por Yachay Wasi (Instituto Superior de Conservación y Restauración) el 30 y 31 de agosto 2013, integrada posteriormente por nuevos avances de la investigación relativos al análisis de la edilicia residencial y de la transformación del tejido urbano. La primera fase de esta investigación ha desarrollado un método de estudio, cuyo objetivo era el reconocimiento del patrimonio arquitectónico en peligro, previo a las intervenciones de recuperación. La segunda fase, actualmente en curso, desarrolla temas conexos a la extensión del concepto de testimonio histórico-arquitectónico y plantea la necesidad de conservación del patrimonio arquitectónico menor, pues contribuye a delinear las características del espacio de la antigua ciudad de Lima. Durante el estudio, se elaboraron unas fichas de análisis que describen de manera completa el valor histórico-artístico y los niveles de riesgo de las estructuras históricas, coordinando datos heterogéneos relativos a la escala arquitectónica y a las relaciones con la ciudad.

\section{PALABRAS CLAVE}

Centro Histórico de Lima, restauración, rehabilitación urbana, fichas de análisis.

\section{ABSTRACT}

The historic center of Lima is a cultural heritage of great interest, currently in an advanced state of decay and in need of an appropriate and effective protection. This article concerns a presentation given in Lima for the "V Jornadas de Conservación y Restauración" organized by Yachay Wasi (Instituto Superior de Conservación y Restauración) on August 30-31, 2013, later implemented by new research advancements on the analysis of residential buildings and urban fabric transformation. Research first phase has developed a methodological approach whose goal was the identification of endagered heritage, prior to every rehabilitation program. Second phase, in course of development, concerns themes related to the widening of the concept of 'cultural heritage', which arises the issue of preserving 'minor' architectural heritage, shaping the features of the space of the old city of Lima. In the study a survey form was developed which fully describes the significance and the level of risk of historic structures, gathering and coordinating highly diverse data related to architectural scale and to relationship with the city. The analysis form is here utilized to assess the conservation of minor architecture with the purpose of proposing an effective rehabilitation strategy and a social and sustainable adaptive reuse

\section{KEYWORDS}

Historic Center of Lima, conservation, urban regeneration, analysis form.

\footnotetext{
1. Este artículo desarrolla con mayor profundidad y detalle las ideas principales de una ponencia presentada en Lima en la V Jornadas de Conservación y Restauración organizadas por Yachay Wasi (Instituto Superior de Conservación y Restauración) el 30 y 31 de agosto 2013 , integrada posteriormente por nuevos avances de la investigación relativos al análisis de la edilicia residencial y de la transformación del tejido urbano.
}

2. Profesora en el College of Architecture, The University of Texas at San Antonio, EEUU. Ha trabajado en la conservación material de la Catedral de Siena, la Catedral de Pienza y en emplazamientos arqueológicos libaneses. Tiene un March, un MS y un doctorado en Restauración Arquitectónica de La Sapienza Universidad de Roma, Italia. (angela.lombardi@utsa.edu).

3. Colaboradora científica en la Historia de la Arquitectura y profesora en el Laboratorio de Historia de la Arquitectura I (Facultad de Ingeniería de L'Aquila) y en la maestría en Mejoramento Sísmico, Consolidación y Restauración de los Monumentos (Universidad de L'Aquila-Universida de Perugia). Doctorado en rehabilitación del patrimonio urbano en La Sapienza Universitad de Roma y una beca de investigación sobre el patrimonio arquitectónico de Avezzano (L’Aquila). 


\section{Lima: Nacimiento y evolución de una ciudad compleja}

Lima es una de las mayores metrópolis de América Latina, así como el centro político, cultural y económico del Perú. En la actualidad, cuenta con más de ocho millones de habitantes e inmigrantes provenientes de todo el mundo, lo que hace de ella una ciudad muy vital y multiétnica. La ciudad posee además un valioso patrimonio histórico arquitectónico constituido por restos arqueológicos, edificios y espacios que datan de los períodos prehispánico, virreinal y republicano, los que en la actualidad se encuentran en su mayoría en grave estado de deterioro y en riesgo de desaparecer.

La ciudad fue fundada oficialmente por el conquistador español Francisco Pizarro, el 6 de enero de 1535, día de la Epifanía, motivo por el que inicialmente se llamó Ciudad de los Reyes, en honor a los Reyes Magos. Posteriormente, fue llamada Lima por el nombre del valle y el río que allí discurría y que la población indígena llamaba Rímac o Límac, que en lengua quechua significa "el que habla", probablemente debido a su cercanía con la Huaca Grande, donde se encontraba el Oráculo del Rímac (El Hablador). En esta zona, existía ya un amplio asentamiento inca, situado en la orilla izquierda del río Rímac y formado por un conjunto de construcciones en tierra cruda o huacas (plataformas, recintos, caminos) entre las que se encontraba la del palacio del curaca o cacique Taulichusco. (Guzmán García, 2012). El nuevo asentamiento se realizó siguiendo un trazado urbano en damero de origen clásico, el trazado hipodámico en el que la plaza principal, la Plaza Mayor, está situada en correspondencia con la huaca de Taulichusco (Mattos Cárdenas, 2012).

Lima, capital del Virreinato del Perú desde el siglo XVII hasta los primeros veinte años del siglo XIX, es durante este periodo una de las ciudades más importantes y ricas de América del Sur y desde un punto de vista arquitectónico, se caracteriza no solo por los importantes monumentos religiosos, sino también por las ricas mansiones señoriales y por las edificaciones menores de valor histórico-arquitectónico relevante. Cabe señalar que aún hoy en día el centro conserva casi intactas, además de la estructura original del trazado fundacional español, las sucesivas ampliaciones llevadas a cabo siguiendo también un trazado urbano hipodámico ortogonal. En efecto, tras la independencia del Virreinato del Perú del Im- perio Español (1821), también la estructura urbana de la ciudad, los sistemas de construcción y las tipologías arquitectónicas virreinales quedaron sustancialmente inalterados durante algunos años.

El cambio, tanto urbano como arquitectónico, empieza a registrarse solo durante el período republicano cuando el aumento de los intercambios comerciales con el extranjero favorece la llegada de inmigrantes, sobre todo desde Europa, y en la arquitectura limeña empieza a notar la influencia europea, en particular del estilo inglés e italiano. Los primeros grandes cambios de la estructura urbana se realizan a partir de la segunda mitad del siglo XIX, por motivos estéticos y funcionales; por ejemplo, se derriban las murallas virreinales y se realiza un nuevo trazado viario anular a lo largo del cual se construyen importantes edificios públicos (1868-1870) y se sobreponen bulevares al tejido urbano virreinal preexistente, siguiendo el modelo de los realizados por Haussmann en París. (Soria León, 2012).

Así, desde la primera mitad del siglo XX, el Centro Histórico de Lima empezó a sufrir no solo transformaciones urbanas y arquitectónicas significativas, sino también sociales. Las familias acomodadas, que tradicionalmente residían en la cuadrícula fundacional renacentista, se transfieren a los modernos barrios de expansión de la periferia, mientras que el centro empieza a acoger grupos de población cada vez más pobres que ocupan y subdividen las ricas residencias unifamiliares del período virreinal y republicano. En 1991, el Centro Histórico de Lima fue nombrado Patrimonio Mundial por la Unesco; sin embargo, en la actualidad un centenar de monumentos y miles de viviendas de valor histórico-arquitectónico se encuentran en estado de abandono y en riesgo de derrumbe (Figura 1 ).

\section{Las fichas de análisis como instrumento de apo- yo para el estudio del patrimonio histórico ar- quitectónico limeño y la definición de directri- ces para su defensa (Angela Lombardi)}

La identificación científica de las fases del proceso de sedimentación cultural en la ciudad histórica se realiza con los instrumentos de la ciencia histórica, indagando y restituyendo críticamente los datos obtenidos con la mayor seguridad posible.

En el caso de los edificios individuales, los complejos edilicios o los contextos arqueológicos deberá llevarse a cabo el recojo de datos de la realidad monu- 


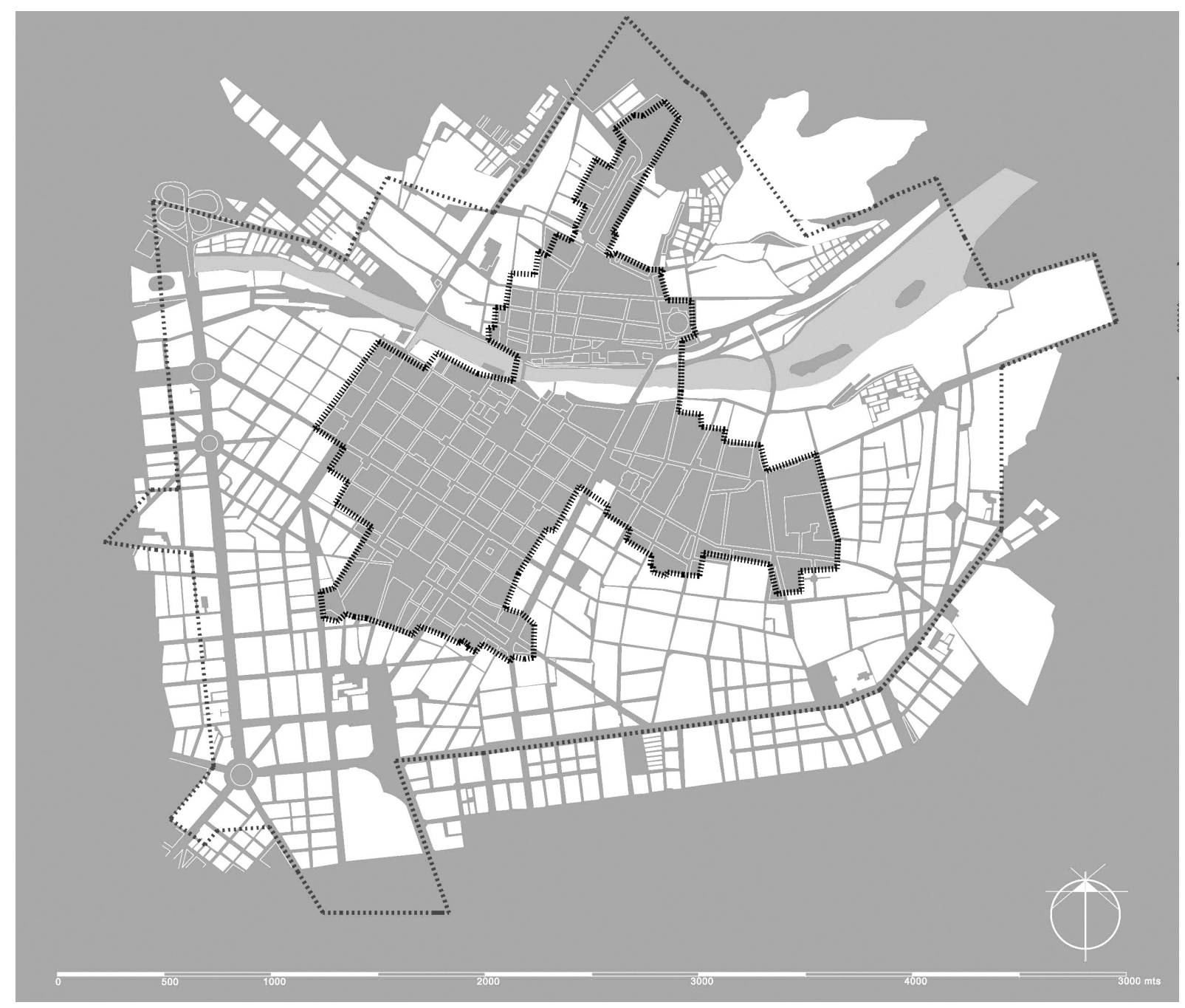

Figura 1. Plano sinóptico del Centro Histórico de Lima con el área declarada Patrimonio Mundial Unesco (línea negra) y la extensión total del Centro Histórico (línea negra). Elaboración. Angela Lombardi. mental desde un punto de vista histórico, artístico y estructural-constructivo documentando además de manera gráfica la entidad actual del conjunto. (Palmerio, 2012).

Durante la investigación conjunta realizada entre La Sapienza Università di Roma y la FAUA de la Universidad Nacional de Ingeniería de Lima, cuyo objetivo era reconocer el patrimonio arquitectónico en riesgo del Centro Histórico de Lima, se llevó a cabo una metodología de análisis que permite un recojo rápido de datos de la realidad monumental gracias a la elaboración de fichas capaces de describir el valor histórico-artístico y los niveles de riesgo de los edificios, reuniendo y coordinando datos muy heterogéneos relativos a los elementos peculiares, las criticidades del edificio y los componentes del contexto urbano y arqueológico. 
Esta metodología fue aplicada, en una versión differente, en el caso de un estudio en profundidad sobre el patrimonio arquitectónico menor y la edilicia residencial del Centro Histórico de Lima en una segunda fase de la investigación desarrollada entre la Università degli Studi dell'Aquila y la University of Texas at San Antonio. De hecho, en la época moderna, muchos edificios residenciales y, en menor modo, numerosos edificios públicos y religiosos del centro de Lima han sufrido intervenciones de alteración tipológica y funcional provocadas por cambios socioeconómicos y urbanos que incluyen el fenómeno de tugurización de los edificios.

La ficha de análisis recoge datos relativos en dos niveles de evaluación: uno relacionado con la estructura analizada, identificando la estratificación, transformaciones, características técnico-constructivas y estado de conservación de las construcciones; el otro, el relativo a las relaciones con el espacio urbano caracterizado por elementos heterogéneos que se refieren a diversos períodos históricos. Los datos recogidos se subdividieron de manera metódica en siete secciones; cada una de las cuales recoge información en grupos de opciones o recuadros con los datos necesarios. (Tabla 1)

Los supuestos teóricos adoptados se valen de los principios expuestos en la Carta de Venecia (1964) y en las Recomendaciones de la Unesco de 1972. La Carta de Venecia introduce el concepto de patrimonio arquitectónico 'menor', ampliando el concepto de 'monumento' a "elementos modestos que han adquirido valor con el tiempo". (art. 5)

Para el procesamiento de los datos, se tuvo en cuenta los estándares internacionales desarrollados para la documentación del patrimonio cultural arqueológico, arquitectónico y mueble (Icomos, 1996), identificando los elementos esenciales de información a incluir en cualquier inventario o documentación de patrimonio cultural. (Icom, 2014).

Los estándares internacionales fueron creados con tal de facilitar el compartir datos más allá de fronteras políticas y para servir de referencia a las organizaciones que trabajan con el patrimonio, las cuales, al crear inventarios del patrimonio, a menudo deben lidiar con la identificación de la colección de datos de inventario con tal de cumplir con los requisitos de administración del patrimonio. La investigación siguió los estándares de documentación más significativos para el inventario del patrimonio arquitec- tónico: se utilizó como referencia el Core Data Index to Historic Buildings and Monuments of the Architectural Heritage, adoptado por el Consejo de Europa en 1992, y el Core Data Standards for Archaeological Sites and Monuments, adoptado por el Centro de Documentación Internacional del Consejo Internacional de Museos en 1995. El Cidoc está finalizando unos estándares combinados para la documentación del patrimonio tanto arqueológico como arquitectónico, conocido como el International Core Data Standard for Archaeological and Architectural Heritage.

La primera sección de la ficha define el tipo de estructura analizada e identifica cuatro tipologías: casa patio, quinta, callejón, otras tipologías.

La casa patio es un tipo de vivienda unifamiliar de origen castellano y es la base del tejido urbano virreinal que encontró en Lima su expresión peculiar; hoy, esta tipología se encuentra a menudo usada para viviendas colectivas o para uso comercial.

Se han identificado dos tipos de complejos residenciales colectivos: el callejón y la quinta. El callejón era un complejo caracterizado por pequeñas casas de una planta, formadas por una habitación y un pequeño patio a los que se accedía desde callejas internas semiprivadas. Los callejones eran habitados, mayormente, por la gente obrera de Lima y por los 'pregoneros' que Ricardo Palma describe en sus Tradiciones peruanas.

La quinta limeña era, en cambio, un conjunto de viviendas edificadas sobre lotes de uso exclusivo, con acceso por un espacio común o directamente desde la vía pública.

La segunda sección enmarca la estructura en el ámbito del contexto urbano en el que se ubica y toma como referencia la Carta de Venecia de 1964, que ratifica la necesidad de salvaguardia no solo de las "creaciones arquitectónicas aisladas", sino también del "entorno urbano y paisajístico" constituido por un tejido urbano menor. Es necesario especificar la época a la que se refiere, las funciones originarias y las funciones actuales. La definición de las funciones

Tabla 1 . Ejemplo de fichas de análisis utilizadas para la catalogación de la arquitectura 'menor' limeña. Elaboración. Angela Lombardi. 


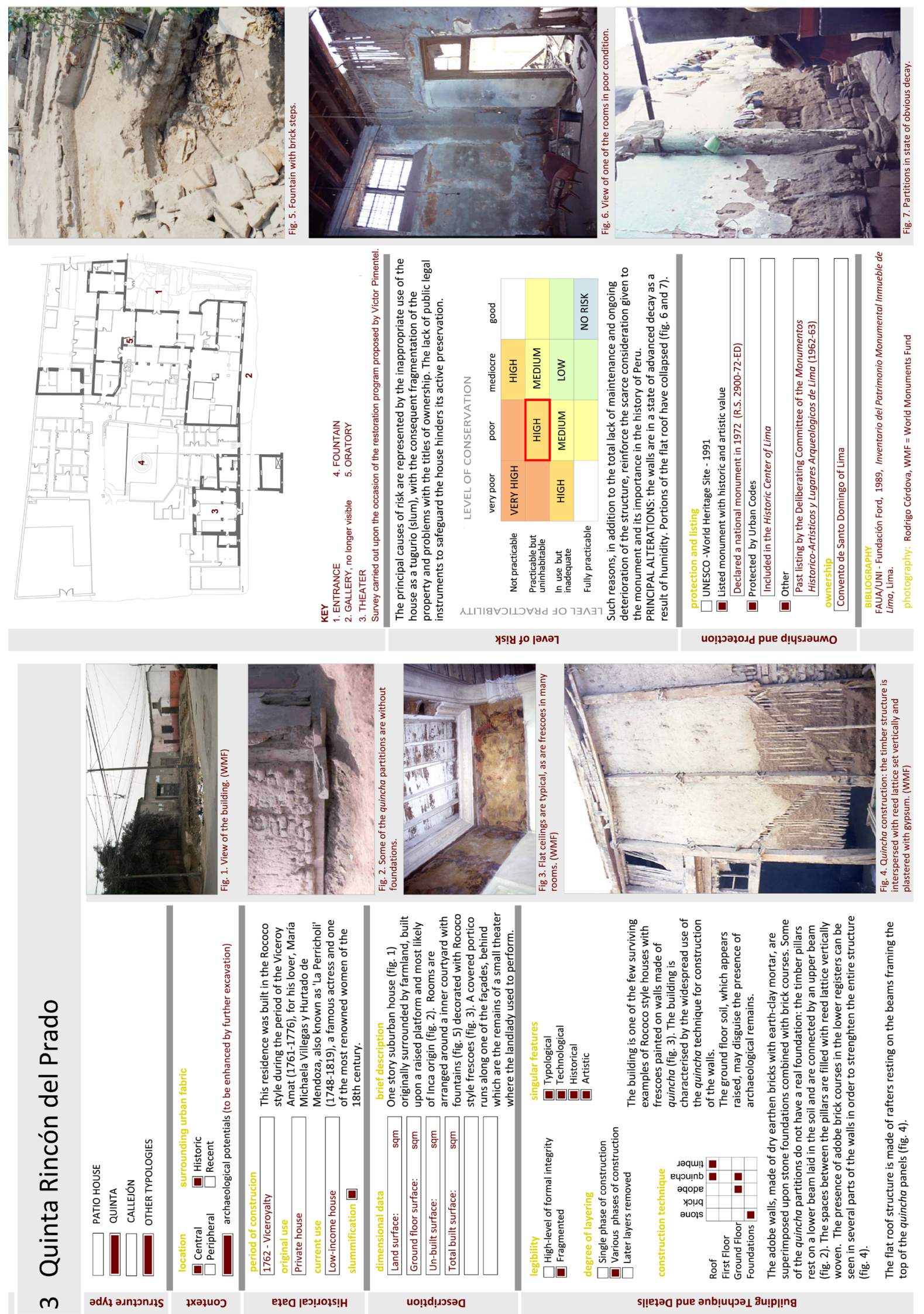


originarias está estrechamente relacionada con el estudio tipológico, de gran importancia localización de la estructura en la ciudad histórica, con referencia al perímetro del Centro Histórico de Lima definido por la Unesco y el tipo de tejido urbano adyacente a la estructura: si este se presenta íntegro en su apariencia virreinal (construcción histórica) o si está colocado en un contexto caracterizado por edificios relativos a diferentes períodos en los que el espacio urbano se caracteriza por el contraste, a veces estridente, entre antiguo y nuevo (nuevas edificaciones), situación recurrente en la ciudad de Lima.

La ficha pretende determinar la presencia de depósitos arqueológicos (incluso por emerger) que darán lugar a excavaciones arqueológicas preventivas, preliminares a toda operación de recalificación. Determinar la presencia de restos arqueológicos (incluso de poca importancia) conllevará a la evaluación de la potencialidad arqueológica del tejido urbano edificado.

La tercera sección contiene los datos históricos relativos al objeto de estudio: el período para el estudio del patrimonio arquitectónico de Lima en relación con las edificaciones menores y con el estudio de los grandes complejos religiosos y civiles. El estudio tipológico, interno a las disciplinas histórica y arqueológica, representa un instrumento fundamental de conocimiento para el arquitecto que actúa en contextos históricos. Por su parte, las funciones actuales, que pueden diferir de las originarias, han producido frecuentes transformaciones del edificio, incluso relevantes si bien a veces no han resultado compatibles con las peculiaridades que lo caracterizan.

La cuarta sección concierne a la descripción de la estructura en la que se incluyen los datos dimensionales y una descripción sintética.

La quinta sección está dedicada al análisis de la técnica constructiva y peculiaridades y está subdividida en cuatro partes: legibilidad, nivel de estratificación, peculiaridades y técnica constructiva.

Con la legibilidad de la estructura, se relacionan dos definiciones: formalmente íntegro o fragmentado, si ha sufrido derrumbes, demoliciones o ha sido objeto de un uso inadecuado, situación frecuente en el caso de Lima donde fachadas aparentemente íntegras se han demolido íntegramente para dejar espacio a nuevas funciones incompatibles con la conservación de la arquitectura histórica, pero que son más rentables respecto de las leyes del mercado.

El nivel de estratificación de un edificio puede referirse a una única fase constructiva o estar constituido por diversas fases constructivas, resultado de la superposición de diversas intervenciones o, en último lugar, encontrarse sin estratificación, si ha sido objeto de operaciones de restauración o de liberación previas que han favorecido la lectura de una fase histórica considerada más significativa.

Las peculiaridades describen los aspectos que caracterizan el objeto de examen y que lo vuelven culturalmente significativo: tipológica, técnico-constructiva, testimonio histórico (en caso de que sea representativo de la historia de la cultura local y/o de la humanidad) o artístico (cuando presente un valor singular o posea representatividad en los elementos arquitectónicos o decorativos que lo caracterizan).

La construcción histórica limeña, de preciadas cualidades arquitectónicas y elementos decorativos de gran importancia, está realizada con técnicas de construcción totalmente originales, resultado de una fusión cultural entre el saber constructivo local y el importado de España. (Figura 3).

La quincha (estructura de listones de madera revestida con un entramado de caña recubierto de barro y un enlucido de yeso) asociada al adobe, (figura 2), gracias a sus características técnicas y su bajo coste se ha impuesto como técnica de construcción tradicional; ha sido utilizada también en las modernas construcciones de principios del siglo XX y solo ha sido sustituida, recientemente, con la llegada del cemento armado. Sin embargo, la quincha aún caracteriza y otorga identidad a la arquitectura de la ciudad y, al mismo tiempo, demuestra la validez de una tradición de construcción de origen prehispánica. Los frecuentes terremotos y sus efectos devastadores, de los que este territorio es continuamente objeto, han demostrado el buen comportamiento sísmico de la quincha. (Soria León, 2012).

Entre los elementos técnico-constructivos más relevantes se encuentra el análisis de los famosos balcones de cajón de Lima que caracterizan, en manera única, la imagen de la Lima virreinal y que introducen un ritmo complejo a la composición de las fachadas urbanas. Estos miradores y balcones encuentran sus orígenes en los musharabíes orientales, pues se remontan a la arquitectura pública del valle del Indo 


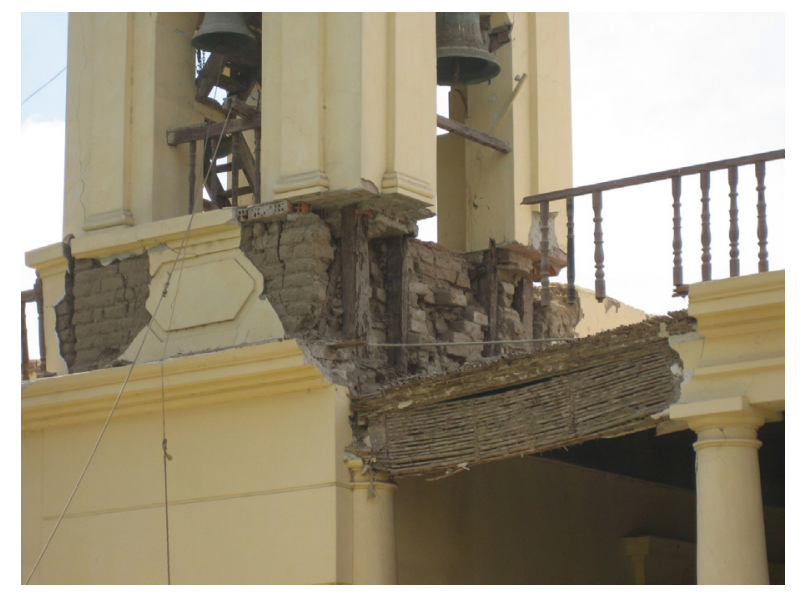

De izquierda a derecha

Figura 2. Formas arquitectónicas barrocas coloniales y técnicas de construcción en quicha y adobe.

Foto. World Monuments Fund. (WMF).

Figura 3. Casa de Manuel Ricardo Palma Soriano. De la casa se conserva parcialmente el cuerpo que da a la calle, con el típico balcón de madera, mientras que en el patio interno unas partes del edificio han sido demolidas.

Foto. World Monuments Fund. (WMF).

y Sumeria, asimilada por los turcos selyaqíes en el siglo XI durante sus continuos contactos con estas tierras y que llegaron a la península ibérica con la dominación árabe (Hurtado Valdez, 2005).

Otro elemento técnico-constructivo de gran interés son los miradores que relacionan la arquitectura limeña con la tradición mudejar. Los miradores son realizados a veces con plantas octogonales y cúpulas conopiales como se encuentra en la preciosa Casa Osambela de inicios del siglo XIX (Olascoaga Mouchard, 2003).

La ficha presenta una matriz que identifica las técnicas de construcción para cada planta del edificio. Se ha dedicado un espacio para la descripción en mayor detalle del uso de cimientos, las propiedades físicas y métricas de los materiales utilizados en las obras murales, etc. Junto a la tecnología constructiva histórica a veces encontramos otras tecnologías modernas y contemporáneas las cuales si están presentes, hay que describirlas de manera específica. La sección, además, está preparada también para la introducción de las planimetrías del edificio y otros posibles diseños técnicos que reflejen aspectos notables.

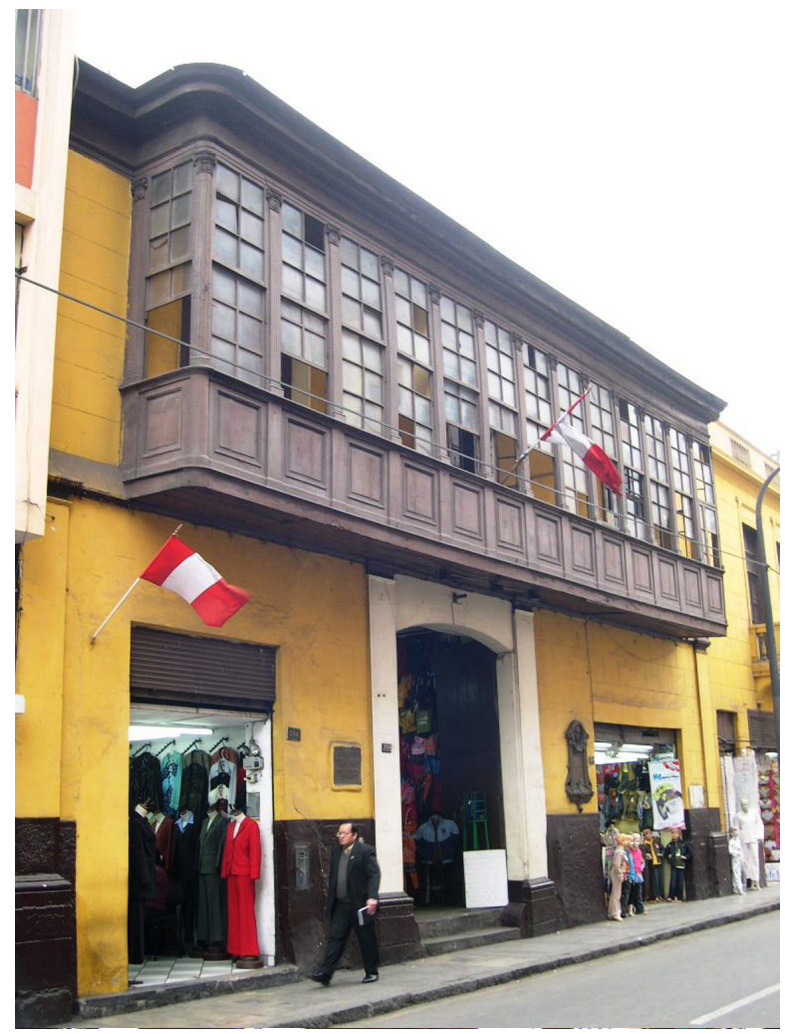

La sexta sección se encarga de identificar el nivel de riesgo al que está sujeta la estructura e introduce los problemas relacionados con su conservación. Amplias zonas del centro histórico de la ciudad se caracterizan por un avanzado estado de deterioro de su patrimonio arquitectónico, con muchos elementos estructurales en riesgo de derrumbe y caracterizados también por su obsolescencia funcional. En la ficha, los niveles de riesgo de una estructura se identifican mediante una matriz formada por dos variables consideradas significativas: la habitabilidad y el nivel de conservación.

La habitabilidad permite valorar el cumplimiento de los requisitos necesarios para garantizar a las personas el uso con seguridad, higiene y salubridad, considerando la adaptación a los estándares higiénicos y de instalaciones, la modalidad de utilización y la compatibilidad de uso respecto a la especificidad histórico-artística. La Declaración de Ámsterdam de 1975 temía el abandono de los edificios que constituyen la ciudad histórica y esperaba que se llevara a cabo un uso compatible con estos, como instrumento activo de conservación. La definición de esta variable toma como referencia teórica los principios ratificados en 1975. (Icomos, 1975).Se han identifi- 
cado, cuatro niveles de habitabilidad: no habitable; utilizable, pero no habitable, en caso que los edificios ofrezcan a los habitantes condiciones de vida y servicios higiénicos precarios, agua potable e instalaciones ausentes o gravemente insuficientes; utilizado, pero no adecuado, con requisitos de seguridad y un moderado confort; utilizado con suficientes requisitos y prestaciones, cuando la estructura cumple perfectamente con los estándares higiénicos y de instalaciones, y mantiene íntegras las peculiaridades que lo distinguen.

El nivel de conservación incluye la evaluación de las condiciones estructurales y del estado de deterioro de los materiales y los elementos técnico-constructivos. Se han identificado cuatro niveles: pésimo, malo, mediocre y bueno.

Por tanto, el nivel de riesgo está definido por las posibles combinaciones de los valores relativos a las dos variables y está representado mediante una matriz en la que se han definido cinco niveles: riesgo muy alto, con necesidad de intervención urgente; riesgo alto, con necesidad de intervenciones de atenuación; riesgo medio, con necesidad de intervenciones de mitigación; riesgo bajo, con necesidad de análisis y exámenes e intervenciones de manutención continua y riesgo nulo, cuando solo es necesaria la manutención ordinaria.

La séptima sección recoge los datos relativos a la propiedad y protección del patrimonio arquitectónico a fin de enmarcar los vínculos, destinatarios y actores del proceso de recalificación; la procedencia de los recursos financieros y los instrumentos de defensa vigentes tanto a nivel local como internacional. Para finalizar, las fichas cuentan con una bibliografía específica para el caso de estudio.

Durante la investigación se identificaron aproximadamente 24 estructuras residenciales, sobre las que se realizaron las fichas (Tabla 1) que son representativas de las diversas tipologías residenciales urbanas y de construcción, además de relevantes por sus elevados niveles de riesgo. La realización de las fichas ha permitido probar su validez, pues se ha revelado una herramienta muy flexible y eficaz, capaz de describir de manera adecuada las más diversas realidades monumentales.

Tras la catalogación de parte del patrimonio histórico-arquitectónico limeño mediante las fichas de análisis descritas, ahora se prevé iniciar una fase su- cesiva del estudio, en la que los datos recogidos se introducirán en una base de datos georeferenciada que se propondrá como proyecto piloto a entidades y autoridades locales.

La finalidad es disponer de una herramienta de documentación e investigación, pero sobre todo de gestión y programación de las intervenciones para la conservación y el desarrollo del centro histórico, conservando intacto su patrimonio residencial. Las fichas y su consiguiente desarrollo en una plataforma SIG representan una herramienta de análisis y conllevan la evaluación de la entidad del patrimonio arquitectónico residencial y de sus condiciones de conservación, además de ser un instrumento indispensable para la definición de las condiciones de riesgo de las construcciones históricas de Lima. Asimismo, son una herramienta de documentación previa al proyecto de restauración del edificio y de su contexto espacial, con el fin de valorizar los componentes peculiares locales y urbanos teniendo en cuenta no solo las edificaciones menores, sino también la población que la habita.

Con el fin de emprender un proceso eficaz de conservación y recuperación del Centro Histórico de Lima, es importante comprender que hoy en día este se caracteriza, como otros centros de América Latina, por la globalidad del patrimonio arquitectónico, por sus espacios urbanos y por el alto nivel de población del centro antiguo causado por el fenómeno de ocupación y subdivisión de las antiguas residencias unifamiliares.

Las fichas de análisis pueden devenir, entonces, en una eficaz herramienta para definir estrategias específicas de "conservación integrada" del patrimonio histórico-arquitectónico limeño e intervenciones específicas dirigidas a la protección y preservación de las estructuras y de los tejidos urbanos antiguos, pero también al saneamiento higiénico-sanitario y a la correcta refuncionalización de los edificios.

\section{Trasformación tipológica de la arquitectura menor del centro histórico de Lima: hacia la ne- cesidad de su conservación integrada. (Patrizia Montuori)}

El Centro Histórico de Lima es un patrimonio cultural de gran interés, constituido no solo por importantes monumentos históricos-artísticos, sino también por el patrimonio arquitectónico 'menor' el cual contribuye a delinear, de forma única y original, las 
características de la ciudad histórica que, sin embargo, se encuentra en precarias condiciones de conservación y necesita de una adecuada y firme acción de protección.

Uno de los aspectos más complejos de las edificaciones menores, en particular aquellas con una finalidad residencial, concierne su progresiva alteración tipológica y funcional mediante intervenciones o finalidades de uso incompatibles así como su conservación.

Con arquitectura menor, se define un conjunto de edificios, principalmente de viviendas, a los que se les reconoce un papel de ambiente de los edificios monumentales y la capacidad de manifestar el carácter de una ciudad a través de elementos típicos, técnicas de edificación y esquemas distributivos que atestiguan una tradición histórico-constructiva. Se hace referencia, por tanto, a esa amplia parte de las construcciones históricas que no están necesariamente ligadas al nombre de un gran autor o a eventos históricos relevantes, pero que sin duda se encuentran en un consolidado equilibrio con la condición de los lugares en los que se ubican y con las que se suele relacionar la imagen de los centros antiguos.

En efecto, en términos teóricos, el concepto de testimonio histórico-arquitectónico por proteger ha sido ampliado desde hace algunas décadas en el ámbito de la conservación. Gustavo Giovannoni ya había ampliado la definición de monumento entendiéndose por este no solo los edificios que se distinguen por su tamaño o sus características histórico-artísticas, sino también "cualquier construcción del pasado, incluso modesta[...] que tenga valor de arte y de testimonio histórico" (Giovannoni, 1945). Por una parte, se ha comprendido que no solo los monumentos son testimonios de civilización, sino que también lo es el patrimonio arquitectónico menor que, en algunos casos, contribuye a delinear las características de la ciudad antigua más de lo que lo hacen las obras expresivas más elevadas. Por otra parte, se ha entendido que únicamente la defensa de los monumentos y la destrucción del contexto histórico arquitectónico causan una grave alteración y una pérdida irreversible de los valores formales y figurativos tanto del edificio en sí como del conjunto.

Por ello, desde hace un tiempo ha madurado la concienciación teórica sobre la necesidad de que toda la ciudad antigua y, en general, todo el entorno construido que revista valor sean objeto de protección.
Sin embargo, permanece aún abierta la cuestión del reconocimiento efectivo de dicho patrimonio como testimonio y a la vez como recurso, además de la reflexión sobre las condiciones actuales y los cambios de uso y de contexto de dicho patrimonio y de los centros históricos.

Efectivamente, en raras ocasiones se pueden mantener en el tiempo las funciones originales de los edificios y de los centros antiguos, y ello a pesar que la introducción de una finalidad de uso inadecuada puede provocar un deterioro muy veloz capaz de destruir o alterar de manera irreversible y en pocas décadas estructuras antiguas muy sólidas.

Del mismo modo, en el caso del Centro Histórico de Lima, uno de los aspectos más complejos concierne a la conservación de las edificaciones menores, en particular aquellas con una finalidad residencial, así como a la progresiva alteración tipológica y funcional de los edificios mediante intervenciones o finalidades de uso incompatibles.

Aún hoy las casas con patios tradicionales de los períodos virreinal y republicano, que en origen se destinaban al uso unifamiliar, son el elemento arquitectónico que más caracteriza al Centro Histórico de Lima. Este patrimonio se compone de ejemplos más ricos y complejos como de edificios más simples, pero igualmente significativos desde un punto de vista histórico-arquitectónico y de asentamiento. La casa situada en el jirón Ica 260, que data quizás en su origen del siglo XVII, fue construida probablemente con el actual estilo Neoclásico tras el terremoto de 1746 y declarada Monumento Nacional en 1974. En cambio, la casa de Manuel Ricardo Palma Soriano, ilustre ensayista y escritor peruano de mediados del siglo XIX, fue construida probablemente en el período virreinal, modificada en el período republicano y declarada Monumento Nacional en 1989. Más simple, pero no menos importante es la casa del siglo XVII situada en el jirón Ancash 935, en los Barrios Altos, donde vivió una de las cantantes más conocidas de la música criolla de la segunda mitad del siglo XX, Lucha Reyes (figuras 3, 4, 5).

De hecho, la casa-patio, tipología de origen castellano que encuentra en Lima una expresión peculiar, es la base del tejido urbano virreinal limeño, basado en un trazado hipodámico ortogonal. Estas casas creaban en el centro de Lima un sistema de espacios similar al de las ciudades antiguas del sur de Europa, 

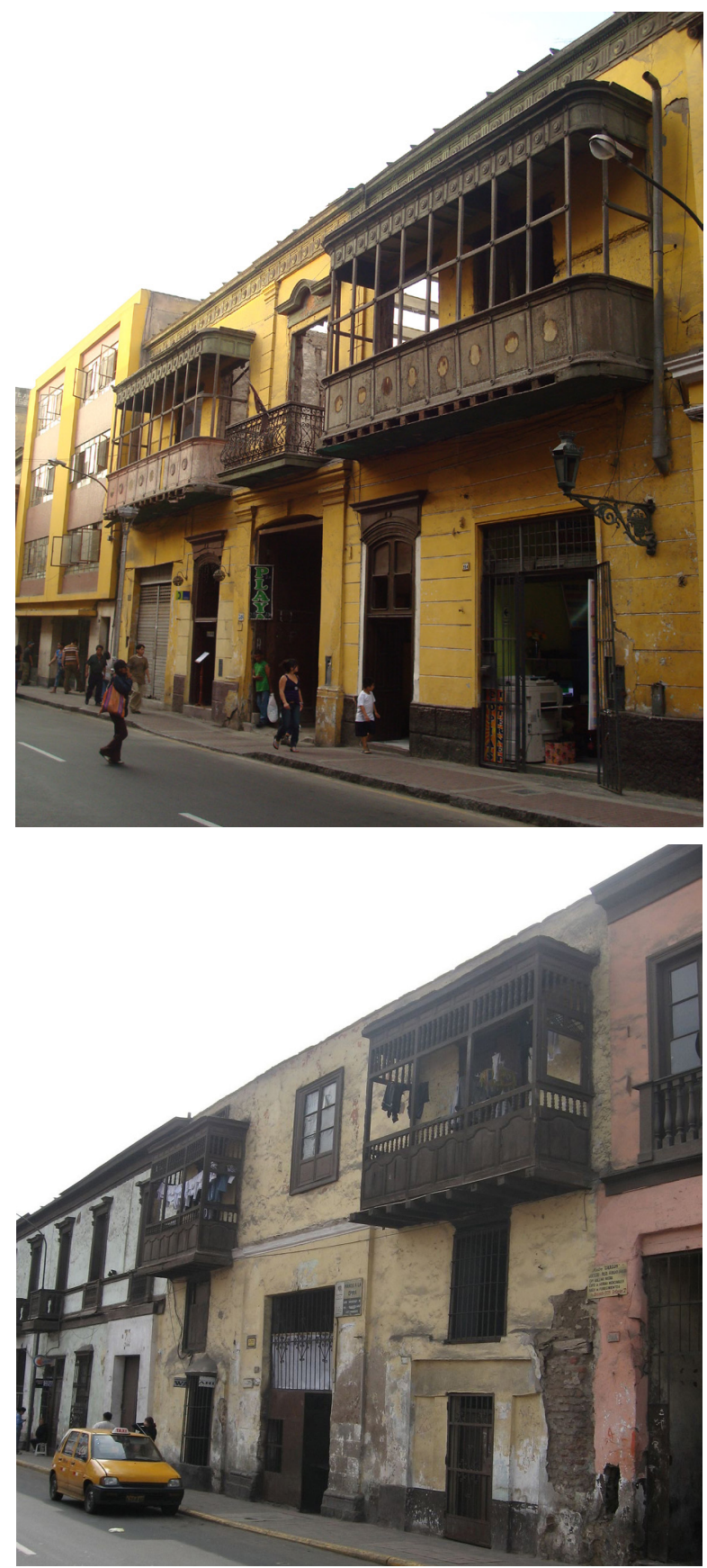

De arriba a abajo

Figura 4. Casa en jirón Ica 260. Vista de la fachada exterior. El interior del edificio fue demolido y del primer piso queda solo la pared perimetral, que sostiene apenas los balcones de cajón.

Foto. World Monuments Fund. (WMF).

Figura 5. Casa en jirón Ancash 935. Esta casa es una lección de historia constructiva en Barrios Altos, y de historia humana: aquí habitó Lucila (Lucha) Reyes, una de las cantantes más importantes de la música criolla. Foto. World Monuments Fund. (WMF). el norte de África y de Oriente Medio, gracias a los patios internos semiprivados abiertos a la calle.

Son también típicos del tejido urbano original de la ciudad los complejos residenciales colectivos, presentes desde la fundación de Lima y probablemente provenientes de los mismos ambientes culturales. Dichos complejos se basaban, por lo general, en un modelo cerrado y, a diferencia de las casas patio, estaban destinados a las clases sociales medio-bajas. Se situaban, por tanto, en las cercanías de edificios productivos donde trabajaban los habitantes o bien en áreas urbanas más periféricas. En Lima, encontramos, por lo general, dos tipos de complejos residenciales colectivos: el callejón y la quinta. El callejón era un complejo caracterizado por pequeñas casas de una planta, formadas por una habitación y un pequeño patio, dispuestas una junto a la otra y a las que se accedía desde callejas internas semiprivadas con hornacinas para santos, placitas y pequeñas capillas donde los habitantes se reunían. La quinta era, en cambio, un tipo de casa colectiva compuesta normalmente por apartamentos, dispuestos uno junto al otro y a los que se accedía directamente desde la calle o, con menos frecuencia, desde una calleja semiprivada. Inicialmente, la quinta tenía una sola planta, pero, tras el aumento demográfico de la ciudad, se añade una segunda planta a los edificios ya existentes y se empiezan a construir quintas directamente de dos plantas, con balcones corridos de acceso y distribución.

En la época moderna, muchos edificios residenciales $y$, en menor modo, los edificios públicos y religiosos del Centro de Lima han sufrido intervenciones de alteración tipológica y funcional provocadas por cambios socioeconómicos y urbanos. En particular, el desplazamiento de las clases medio-burguesas desde el trazado fundacional hacia los nuevos barrios de expansión ha tenido efectos destructivos tanto en el centro como en los edificios históricos.

Las antiguas residencias unifamiliares de los períodos virreinales y republicanos fueron subdivididas sin criterio y ocupadas por diversos núcleos familiares pertenecientes a clases sociales medio-bajas. Los edificios suelen estar en grave estado de deterioro, debido también a la imposibilidad económica de los habitantes de realizar intervenciones de manutención y restauración o de adecuación funcional e higiénicosanitaria. Dicho proceso de tugurización incluye también a los complejos residenciales colec- 
tivos, más adecuados tipológicamente para albergar a diferentes familias, y que, al mismo tiempo, están en precarias condiciones de conservación por la falta de manutención debida a los escasos recursos económicos de los habitantes.

Este drástico descenso del nivel social de los habitantes ha causado también el desplazamiento de las funciones directivas hacia la parte sur de la ciudad, mientras que el centro se ha caracterizado progresivamente como lugar de venta ambulante ilegal. Muchos edificios históricos han sufrido transformaciones inadecuadas, debido al aumento de la actividad comercial y han sido transformados en pequeñas empresas o tiendas. Las aceras del centro han sido ocupadas por los puestos de los vendedores ambulantes que impiden la vista de los edificios históricos, las calles y las plazas y a menudo causan también daños en las fachadas. Después de la designación de Patrimonio de la Humanidad, el gobierno municipal ha intentado expulsar del centro histórico a los vendedores ambulantes mediante normativas, planes e incluso con la ayuda de las fuerzas policiales y de gases lacrimógenos. Sin embargo, la oposición a estas intervenciones ha sido muy fuerte y los resultados, bastante decepcionantes (Säppanen, 1999).

En general, parece escasa la percepción del valor histórico-arquitectónico del centro de Lima, pues está considerado simplemente un lugar económicamente ventajoso por la abundancia de demanda, donde se concentran intereses de tipo especulativo. Muchos edificios de valor histórico-arquitectónico han sido total o parcialmente destruidos de manera intencionada a fin de instalar aparcamientos y actividades comerciales o para construir nuevos edificios. Hoy en día, en la planta baja de la casa situada en el jirón Ica 260 queda solo el zaguán y algunas habitaciones que sirven de tiendas, mientras que en el primer piso solo se conserva la pared perimetral que sostiene a duras penas los típicos balcones de cajón. Incluso la misma Municipalidad de Lima, tras la destrucción de los volúmenes originales, ha instalado allí un aparcamiento. Muy significativo es también el derumbe del edificio El Buque, causado por un incendio probablemente intencionado. El edificio databa del siglo XVIII y era el único de tres niveles situado en los Barrios Altos, con la planta baja construida en adobe y las superiores, en quincha. Es probable que, originalmente, se tratara de una amplia casa patio, transformada posteriormente en un complejo residencial con pequeños apartamentos a los que se accedía desde el balcón corrido al exterior del tercer piso.(figuras 6).

En realidad, a partir de 1991 y, en particular, tras ser declarado el Centro Histórico de Lima Patrimonio de la Humanidad, se han realizado, gracias a inversión pública y privada, intervenciones principalmente de embellecimiento con una finalidad turística: nueva pavimentación, mobiliario urbano e iluminación de espacios públicos o pintado de los edificios. Poco sustancial ha sido lo realizado por la defensa del patrimonio histórico-arquitectónico y por la puesta en marcha de intervenciones de recuperación adecuadas, sobre todo de los edificios residenciales en estado de deterioro.

En dicho contexto, es particularmente interesante la intervención de consolidación, restauración y adaptación higiénico-sanitaria de la Casa de las Columnas realizada por diversas organizaciones nacionales e internacionales entre 2008 y 2010.

Esta edificación, que en 1972 fue declarada Monumento Nacional, era originalmente una parte del Convento de Nuestra Señora del Rosario, uno de los primeros fundados en Lima a finales del siglo XVI y destruido por el terremoto de 1746. El convento, formado por ocho claustros y patios, ocupaba dos cuadras de la calle Conde de Superunda y distaba una cuadra de la Plaza de Armas, hoy en día conocida como Plaza Mayor. El edificio se encontraba en condiciones de conservación precarias debido también a que, al igual que otros edificios deshabitados del centro histórico, había sido ocupado por familias con escasos recursos financieros y subdividido en varias viviendas, sin la adaptación higiénico-sanitaria necesaria. Tras la inclusión de la Casa de las Columnas en la lista de 2008 del World Monuments Watch, se puso en marcha un proyecto de recalificación y restauración del edificio, así como de formación de trabajadores especializados gracias a una Escuela-Taller. El aspecto más interesante de la intervención es, sin duda, el que el edificio no fuera considerado un mero recurso económico en el que realizar intervenciones de demolición, modificación de finalidad de uso o embellecimiento con finalidad turística. Por el contrario, la Casa de las Columnas fue considerada un complejo residencial de valor histórico-arquitectónico que debía seguir sirviendo a las necesidades de sus habitantes. Por ese motivo, se comprendió que el precario estado de conservación de la estructura requería, en primer lugar, intervenciones cuidado- 


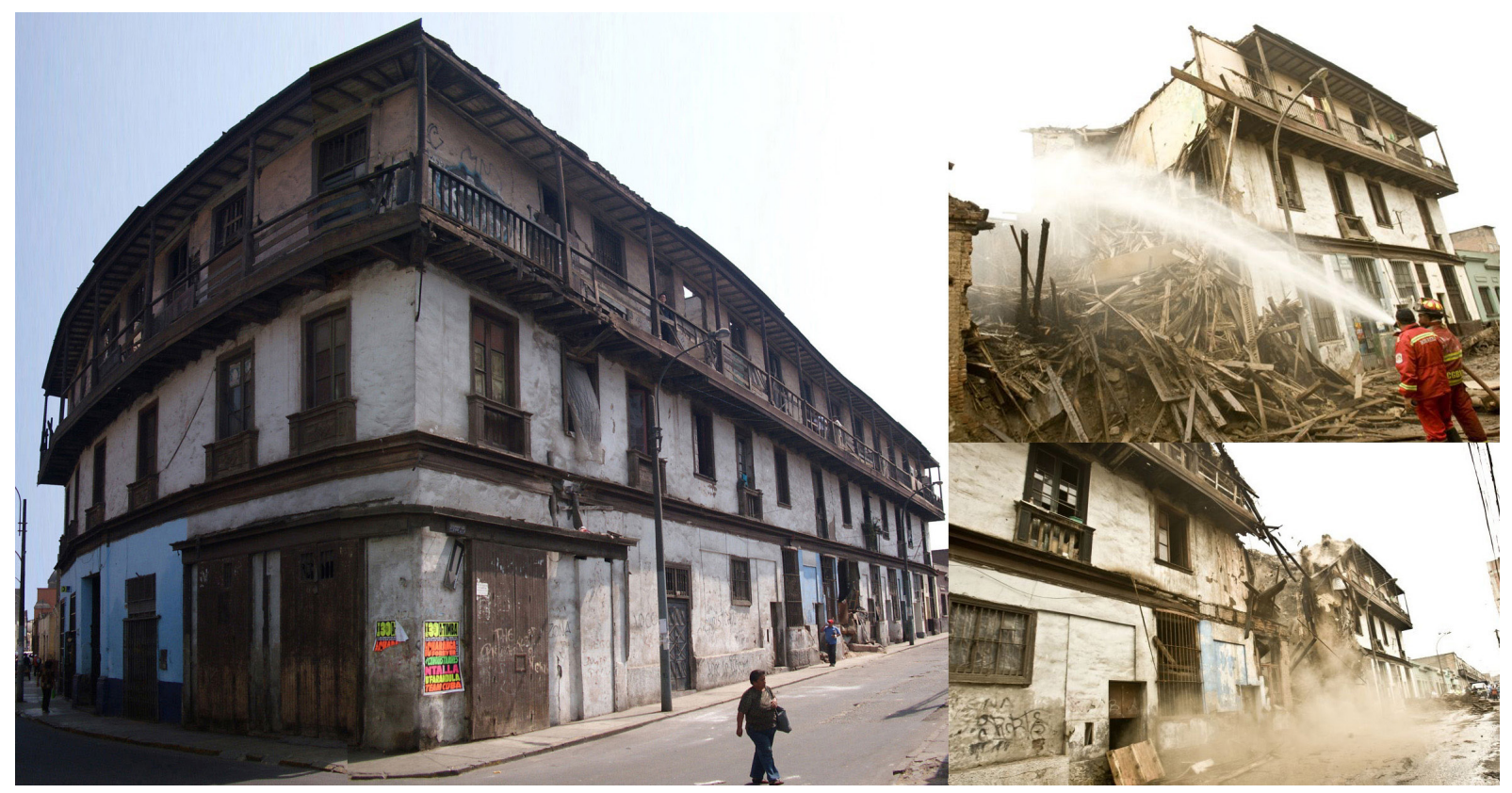

Figura 6. Edificio El Buque antes y después del colapso. En noviembre de 2012, un incendio causó el colapso de esta mansión histórica construida en 1753 y nominada Monumento Nacional en 1988 .

Foto izquierda en www.welovetours.blogspot.com; fotos derecha: Karen Zárate / El Comercio en http://elcomercio.pe/actualidad/1492241/noticia-fotos-asi-quedo-viejo-solar-buque-afectado-incendio-derrumbe

sas con el fin de garantizar su estabilidad estructural, además de adecuados niveles de seguridad e higiene para las numerosas familias que allí residen. Por ejemplo, la instalación de nuevos servicios comunes supuso una mejoría esencial para los residentes, ya que los inmuebles no contaban con servicios higiénicos privados. Estas nuevas instalaciones han contribuido a mejorar la higiene y han transformado las zonas comunes del edificio en un espacio de encuentro para los inquilinos.

Esta intervención demuestra que para iniciar un proceso de conservación y recuperación adecuado y eficaz del Centro Histórico de Lima es importante, en primer lugar, comprender que dicho centro, al igual que otros de América Latina, hoy en día se caracteriza no solo por los edificios y espacios urbanos de valor histórico-arquitectónico, sino que la numerosa población causada por el fenómeno de ocupación y subdivisión de las antiguas residencias unifamiliares e, incluso, la amplia difusión de actividades comerciales si bien son causa clara de problemas y deterioro, también son ya una parte integrante de su imagen y su orden económicosocial. Los efectos negativos de dichos fenómenos deberían mitigarse con intervenciones orgánicas de conservación, restauración y adaptación funcional de los edificios que no desvirtúen con una finalidad exclusivamente turística la esencia del centro y los edificios históricos de Lima y que sean realmente eficaces. Por lo tanto, el tema central es la definición de estrategias de intervención específicas y complejas que contemplen la defensa y protección de las construcciones y tejidos urbanos antiguos además del saneamiento higiénico-sanitario de los edificios y su adecuada refuncionalización

Este enfoque es totalmente compatible con el concepto de conservación integrada, el cual se propuso por primera vez en la Carta Europea del Patrimonio Arquitectónico, promulgada en Amsterdamen 1975, entendida como "el resultado de la acción conjunta de las técnicas de restauración y el estudio de funciones compatibles que realizar junto con una puesta a punto bien efectuada de los medios jurídicos, administrativos, financieros y técnicos". Gracias a este enfoque la conservación y uso adecuado de los edificios históricos no obtendría, así, importantes resultados solo desde el punto de vista cultural, sino que podría 
mejorar el nivel de vida de los habitantes del Centro Histórico de Lima y desencadenar un desarrollo económico inducido por estas intervenciones a realizar.

Agradecimientos: Quisiéramos dar las gracias al profesor Leonardo Mattos-Cárdenas por sus valiosos consejos; al profesor Giancarlo Palmerio, nuestro coordinador en el estudio realizado en la Universidad Sapienza de Roma; a los profesores Rodrigo Córdova y Judith Soria y, en último lugar, pero no por ello menos importante, al World Monuments Fund.

\section{Bibliografía}

Carbonara, G. (1996). Avvicinamento al restauro. Napoli: Liguori.

Giovannoni, G. (1945). Il restauro dei monumenti. Roma: Cremonese.

Guzmán García, E. (2012). History and pre-Hispanic Traces in the Viceroyal Plan of Lima. En G. Palmerio, A. Lombardi \& P. Montuori, (Eds). Lima. The Historic Center. Analysis and Restoration, pp.24-29. Roma: Gangemi Editore.

Mattos Cárdenas, L. (2012). Viceroyal Lima. Origins and Development of a Complex City. En G. Palmerio, A. Lombardi \& P. Montuori, (Eds). Lima. The Historic Center. Analysis and Restoration, pp.19-23. Roma: Gangemi Editore.

Olascoaga Mouchard, J. F. (2003), Desarollo histórico del conjunto arquitectónico de Santo Domingo de Lima. Lima: (s.n.)

Palmerio, G. (2012). Identification and Preservation of the Architectural Heritage of the Historic City. En G. Palmerio, A. Lombardi \& P. Montuori, (Eds). Lima. The Historic Center. Analysis and Restoration, pp. 42-47. Roma: Gangemi Editore.

Säppanen, M. (1999). Global scale, Local Place? The Making of the Historic Centre of Lima into a World Heritage Site. Helsinki: Institute of Development Studies, Interkonts Book.

Soria León, J. (2012). The New Face of the City between the 19th and the 20th Century. En G. Palmerio, A. Lombardi \& P. Montuori, (Eds). Lima. The Historic Center. Analysis and Restoration, pp. 30-35 Roma: Gangemi Editore.

Soria León, J. (2012). Traditional Construction Techniques of Lima. Materials and Systems. En G. Palmerio, A. Lombardi \& P. Montuori, (Eds). Lima. The Historic Center. Analysis and Restoration, pp.106-111. Roma: Gangemi Editore.
World Monuments Fund (2012). Casa de Las Columnas - Social Rehabilitation through Monument's Restoration. En G. Palmerio, A. Lombardi \& P. Montuori, (Eds). Lima. The Historic Center. Analysis and Restoration, pp. 98-105. Roma: Gangemi Editore.

Hurtado Valdez, P. (2005). Entre torres y balcones: la imagen de la Lima Virreinal. Areté Documenta, (20), Recuperado de http://oa.upm.es/4969/1/Entre_Torres_y_Balcones.pdf [consultado el 20/07/2014].

Icomos. (1964). International Charter for the conservation and restauration of monuments and sites. The Venice Charter 1964. Recuperado de http://www.icomos.org/ charters/venice_e.pdf

Icomos. (1975). The Declaration of Amsterdam-1975. Recuperado de: http://www.icomos.org/en/chartersand-texts/179-articles-en-francais/ressources/charters-and-standards/169-the-declaration-of-amsterdam

Icomos. (1996). Principles for the recording of monuments, groups of buildings and sites. Recuperado de: http:// www.icomos.org/en/179-articles-en-francais/ressources/charters-and-standards/387-principles-for-the-recording-of-monuments-groups-of-buildings-and-sites-1996

Icom. (2014). Core Data Index to Historic Buildings and Monuments of the Architectural Heritage, 2014. Recuperado de: http://archives.icom.museum/objectid/ heritage/intro3.html .

Unesco. (1972). Recommendation concerning the Protection, at National Level, of the Cultural and Natural Heritage. Recuperado de: http://portal.unesco.org/en/ev.php-URL ID $=13087 \&$ URL_DO=DO_TOPIC\&URL_SECTION $=201$. html 